\section{Ultrasonography of benign vulvar lesions}

\author{
Vincent Y. T. Cheung \\ Department of Obstetrics and Gynaecology, Queen Mary Hospital, The University of Hong \\ Kong, Hong Kong
}

With the increasing applications of ultrasonography in the field of dermatology, this imaging modality can be extended to identify and characterize common vulvar lesions, with a particular focus on their location, size, vascularity, and content. This pictorial essay provides an introduction to vulvar ultrasonography, including technical considerations, ultrasonographic anatomy, and the ultrasonographic appearances of some vulvar conditions.

Keywords: Ultrasonography; Vulva; Vulval ultrasound

\section{Introduction}

With improvements in ultrasound technology, particularly in the development of a newer generation of machines that work with high- and variable-frequency probes, ultrasonography has become increasingly useful for imaging superficial structures. Ultrasound has the capability of distinguishing skin layers, and therefore, can be used as a tool to evaluate cutaneous pathologies. In recent years, the applications of ultrasonography in dermatology have been growing $[1,2]$. The vulva, which consists of the mons pubis, labia majora, labia minora, clitoris, and the vestibule containing the urethral meatus and the vaginal opening, can be affected by a wide variety of dermatological conditions, some of which are unique to this area. The application of high- and variable-frequency ultrasound increases the degree to which common vulvar lesions can be identified and characterized with regard to their location, size, vascularity, and content. To our knowledge, very few articles in the literature have described the clinical applications of ultrasound in evaluating vulvar lesions, except in relation to the assessment of the supporting structures, incontinence, and pelvic organ prolapse. This pictorial essay serves as an introduction to the relevant sonographic techniques and the normal anatomy of the vulvar skin, and provides an illustration of certain vulvar entities that the author has recently encountered and evaluated sonographically. This overview will help promote physicians' interest in using ultrasound on the vulva, with the ultimate aim of evaluating whether this imaging modality can be clinically applicable for the evaluation of common vulvar conditions.

\section{Scanning Techniques}

Ideally, as in other dermatologic applications, high- and variable-frequency transducers working with upper frequencies of $15 \mathrm{MHz}$ or higher are recommended [1]. A lower-frequency transducer can be used, but the definition of skin layers may be suboptimal. The scanning techniques for the vulva are similar to those that have been described for skin lesions on other parts of the body [1]. However, the vulva is in a peculiar location and acoustic gel can slide off quickly despite multiple applications.

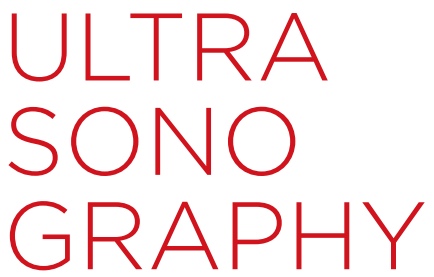

PICTORIAL ESSAY

https://doi.org/10.14366/usg. 18001 pISSN: 2288-5919 • elSSN: 2288-5943 Ultrasonography 2018;37:355-360

Received: January 1, 2018

Revised: February 5, 2018

Accepted: February 13, 2018

Correspondence to: Vincent Y. T. Cheung, MBBS, FRCOG, FRCSC, Department of Obstetrics and Gynaecology, Queen Mary Hospital, The University of Hong Kong, 102 Pokfulam Road, Hong Kong

Tel. $+852-22553914$

Fax.+852-25173278

E-mail: vytc@hku.hk

This is an Open Access article distributed under the terms of the Creative Commons Attribution NonCommercial License (http://creativecommons.org/ licenses/by-nc/3.0/) which permits unrestricted noncommercial use, distribution, and reproduction in any medium, provided the original work is properly cited.

Copyright (C) 2018 Korean Society of Ultrasound in Medicine (KSUM)

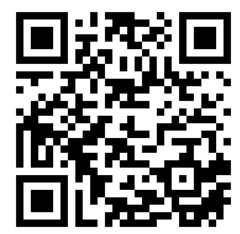

How to cite this article: VYT Cheung. Ultrasonography of benign vulvar lesions. Ultrasonography. 2018 0ct;37(4):355360. 
Scanning needs to be quick with occasional gel replacement over the involved area. No or only minimal pressure should be applied on the skin to avoid distortion of the vulvar structures. The greyscale examination of a lesion should be carried out in at least two perpendicular planes, followed by color Doppler imaging if necessary. If the target lesion is identified, an evaluation of the echogenicity patterns, measurement of its size, identification and measurements of the blood flow (distribution, type, and maximum peak systolic velocity of the arterial vessels), and localization, particularly in relation to the skin layers, should all be reported.

All images shown in this article were obtained using the Samsung Medison Accuvix XG System (Samsung Medison Co., Ltd., Seoul, Korea) with a variable linear transducer with an upper frequency of $13 \mathrm{MHZ}$.

\section{Sonographic Anatomy}

The skin is composed of three layers: the epidermis, dermis, and hypodermis (also known as subcutaneous tissue). On 2-dimensional greyscale sonography, the echogenicity of each layer depends on its main component: keratin in the epidermis, collagen in the dermis, and fat lobules in the subcutaneous tissue [1]. The epidermis usually appears as an echogenic line (Fig. 1) [1]. However, because the vulvar skin has various degrees of keratinization, from keratinized epithelium on the labia majora to non-keratinized epithelium on the labia minora, the echogenicity of the epidermis on the vulva can vary. In areas with a thicker corneous layer, such as the medial aspect of the labia minora, the epidermis may appear as a bilaminar parallel hyperechoic structure (Fig. 1).

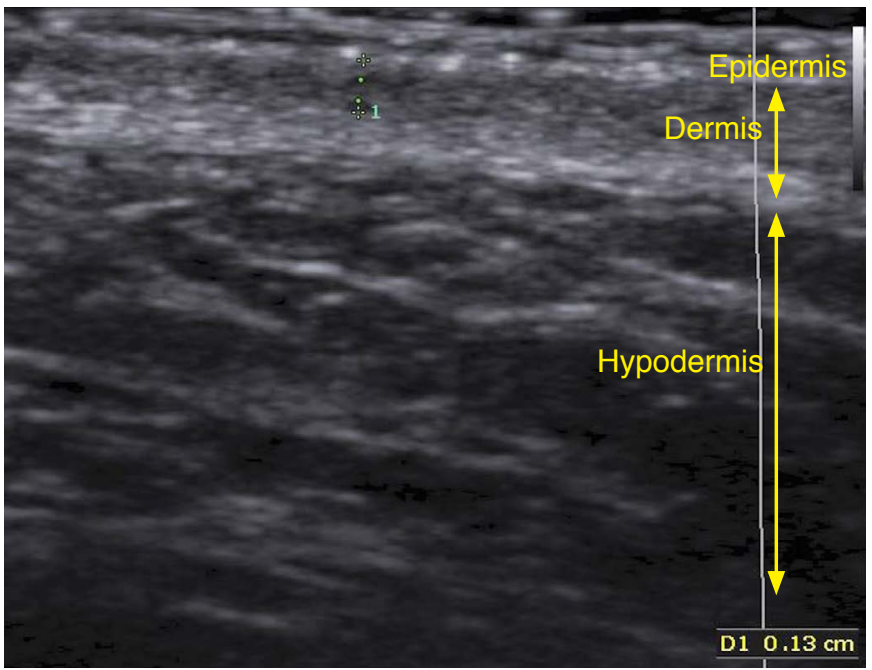

Fig. 1. Sonographic anatomy of the vulvar skin. From a 45-yearold woman, this image was taken near the labia minora, where the epidermis appeared as a bilaminar parallel hyperechoic structure.
The dermis appears as a hyperechoic band, less bright than the epidermis (Fig. 1). As the collagen content in the vulva can vary, particularly depending on the patient's age, the echogenicity and thickness of the dermis can also be variable [3]. The identification of this layer is also difficult, as the vulvar structures are compacted and the overlying skin is generally wrinkled. Beneath the dermis, fatty lobules provide the main source of echogenicity of the hypodermis, which is mostly hypoechoic and contains hyperechoic strands of fibrous septa (Fig. 1).

Three-dimensional volume reconstruction and color and/or power Doppler ultrasound can be performed selectively to improve the image or highlight the vascularity.

\section{Vulvar Lesions}

\section{Bartholin Cyst and Abscess}

Bartholin cyst is the most common cystic lesion of the vulva, and arises from cystic dilatation of an obstructed Bartholin duct. This cyst is usually seen as a unilateral, fluctuating structure at the posterior third of the labia majora and contains clear mucous fluid. Sonographically, this cyst is located in the hypodermis with intact epidermis and dermis. It appears mostly anechoic to hypoechoic due to predominant fluid content, with a well-defined cyst wall. Echo enhancement is frequently seen posterior to the cyst due to increase through transmission of the cyst content (Fig. 2) [4].

When infected, the overlying skin appears red and becomes

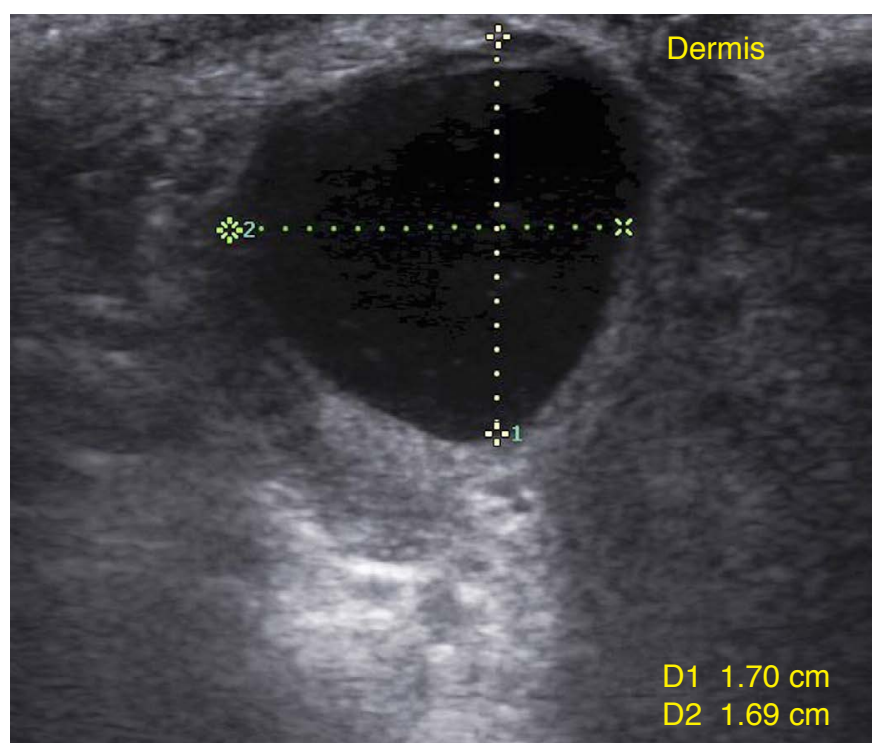

Fig. 2. A 44-year-old woman presented with a right Bartholin cyst. Sonographically, this cyst was well-defined, mostly anechoic with acoustic enhancement, and was located in the hypodermis with intact epidermis and dermis. 
tender on palpation. The cyst wall contains pus instead of clear fluid, and the sonographic appearance is a collection of echogenic fluid, occasionally containing septa and sediment. The cyst wall may become echogenic with less well-defined borders due to the inflammatory response of the adjacent tissue (Fig. 3) [5].

\section{Epidermal Cyst}

An epidermal cyst is derived from the entrapment of epidermal components in the dermis and hypodermis (Fig. 4A). On ultrasonography, these cysts appear as well-defined, oval or round, anechoic or hypoechoic structures located in the dermis and hypodermis, frequently with a posterior acoustic enhancement artifact (Fig. 4B).

\section{Lipoma}

Although lipomas are common benign tumors of the soft tissue, their occurrence in the vulva is uncommon. The ultrasonographic appearance of vulvar lipomas has been described as whorl-shaped, lobular structures that do not show vascularization on color and power Doppler imaging (Fig. 5) [6].

\section{Hemangioma}

Resulting from the abnormal proliferation of blood vessels,

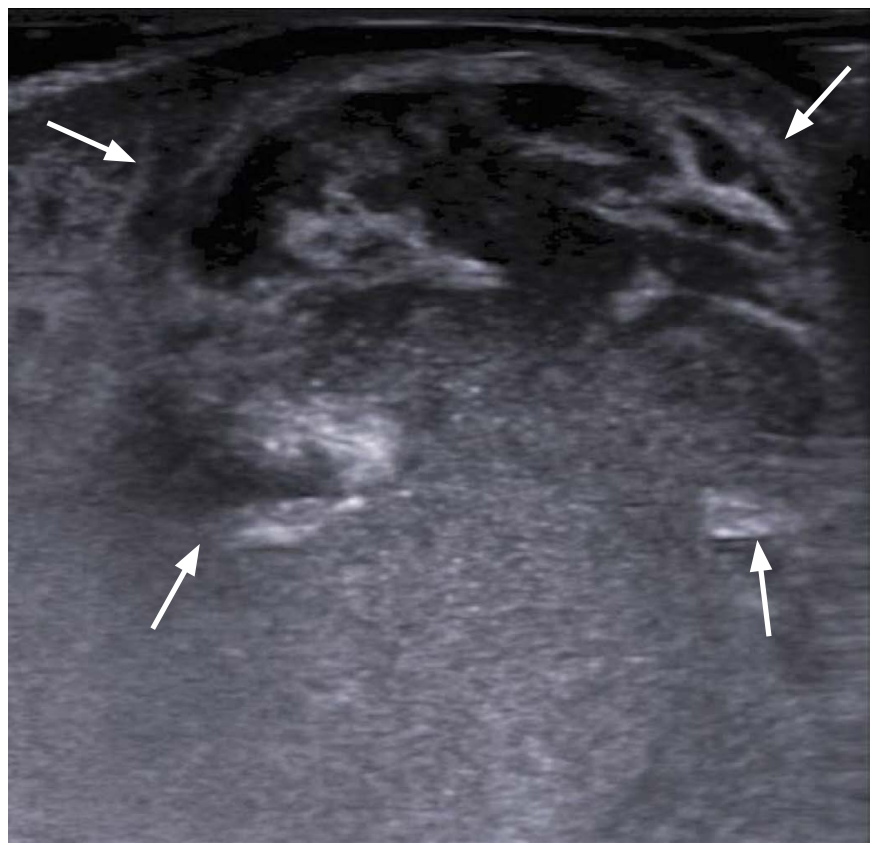

Fig. 3. A 31-year-old woman presented with a tender left vulvar swelling, clinically diagnosed as a left Bartholin abscess. On ultrasonography, the mass appeared as a collection of echogenic fluid with occasional septa and sediment. The cyst wall may become echogenic with less well-defined borders. The arrows indicate the outline of the abscess.

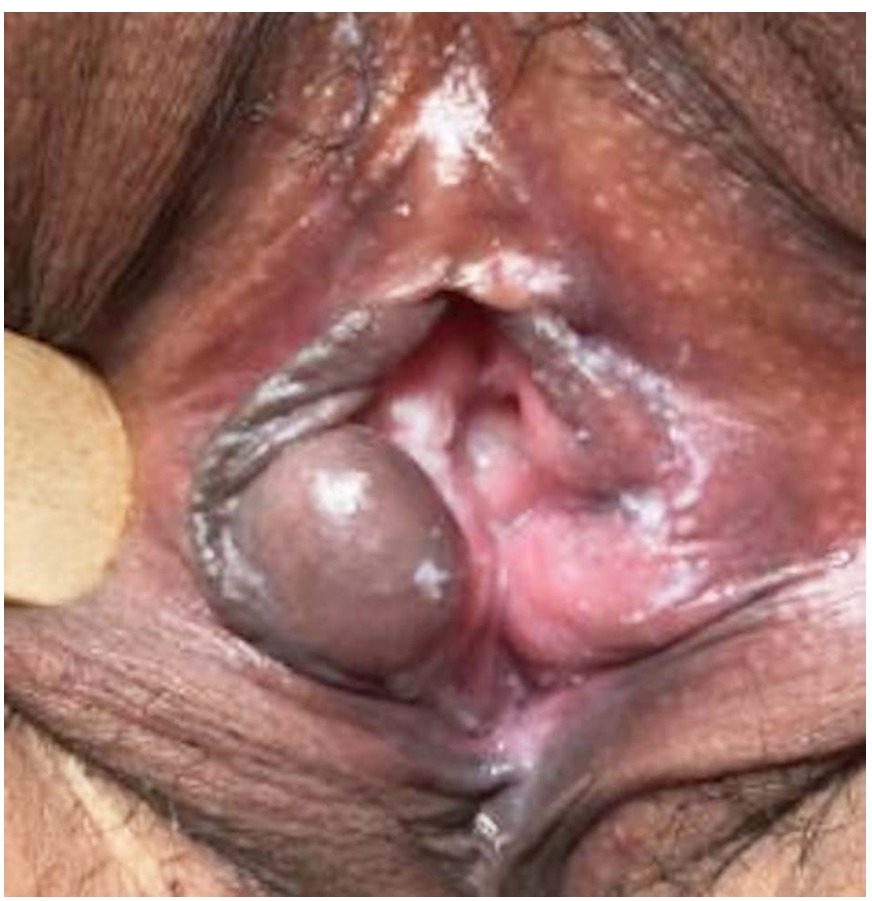

A

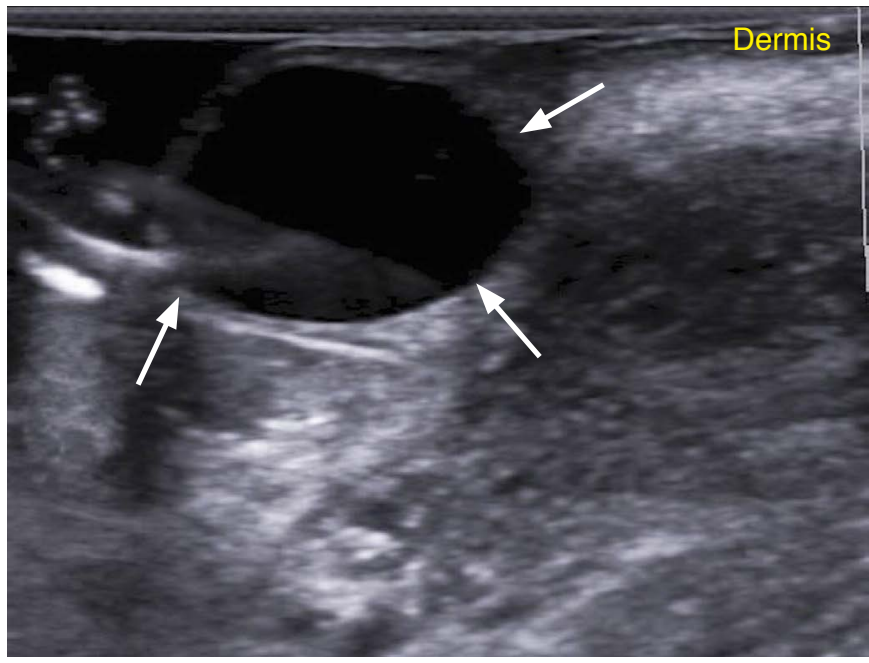

B

Fig. 4. A 49-year-old woman presented with a right epidermal cyst.

A. Clinical image shows the bulging contour of the right paramidline vulvar lesion. B. Ultrasonography shows a $1.2 \times 0.9-\mathrm{cm}$ well-defined, anechoic cyst (arrows) located in the dermis and hypodermis. 


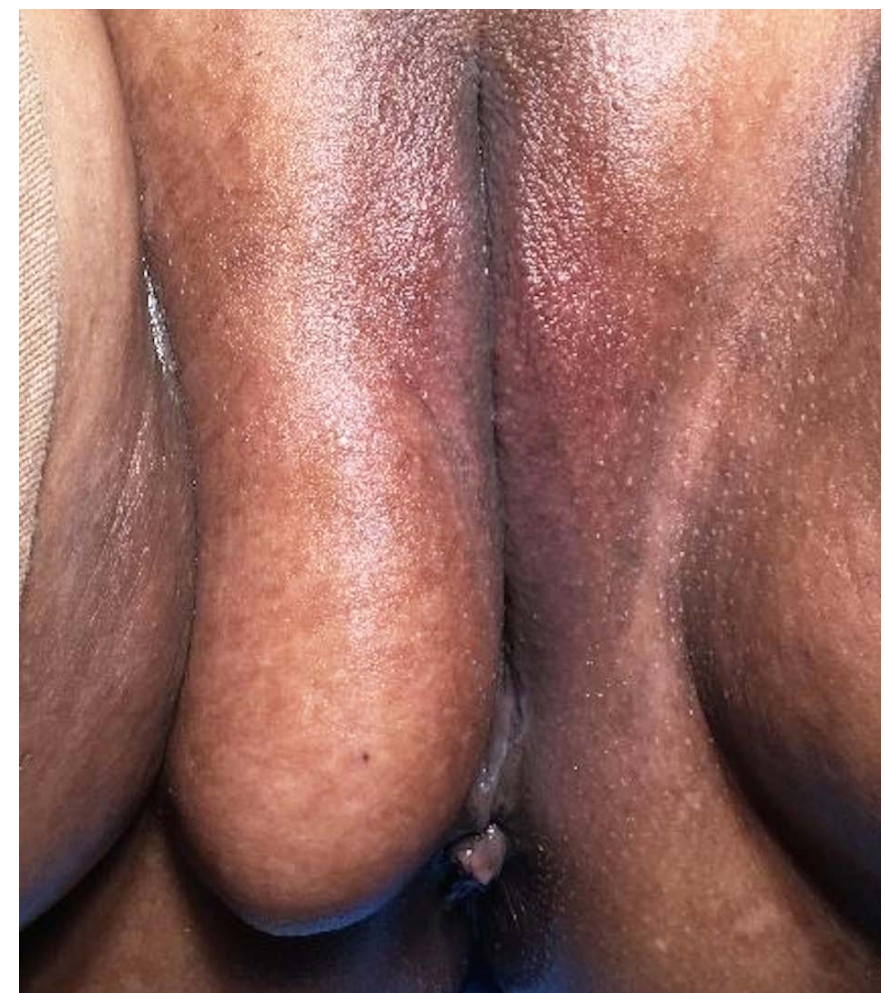

A

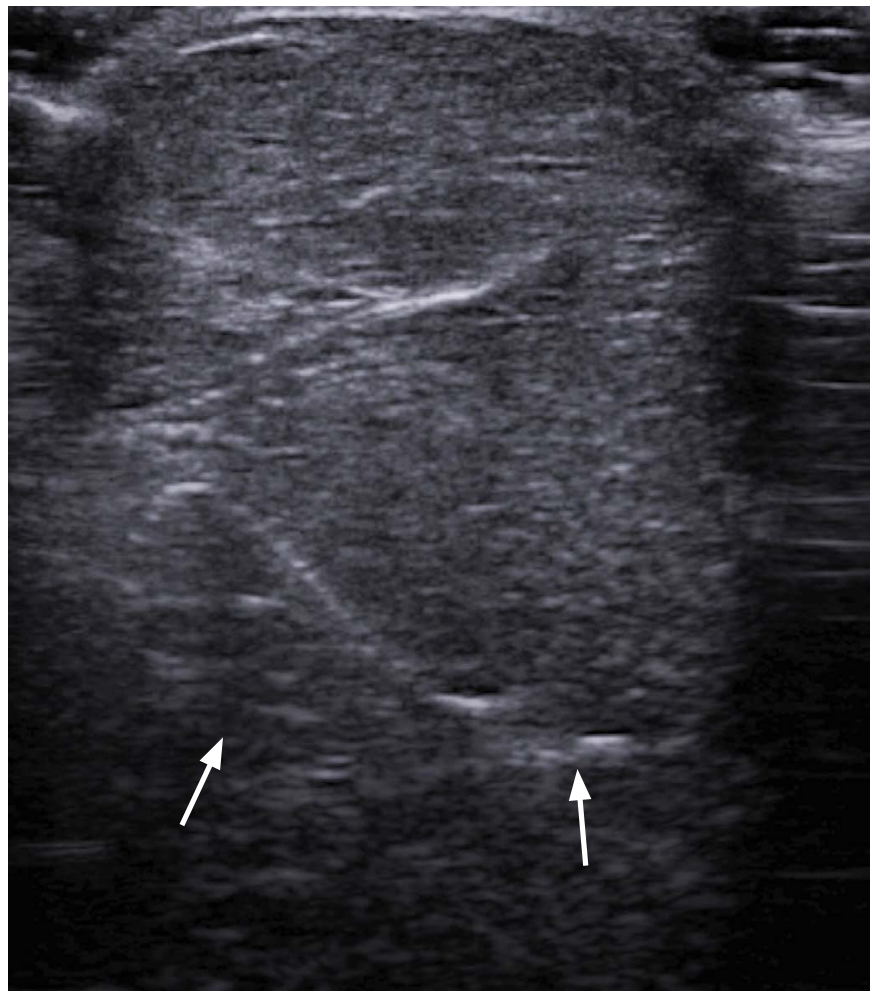

B

Fig. 5. A 33-year-old woman presented with a right vulvar lipoma, confirmed histologically on excisional biopsy.

A. Clinical image shows the bulging appearance of the lipoma (image provided by courtesy of Dr. Charleen Cheung). B. Ultrasonography shows a whorl-shaped, lobular mass. The arrows outline the depth of extension.

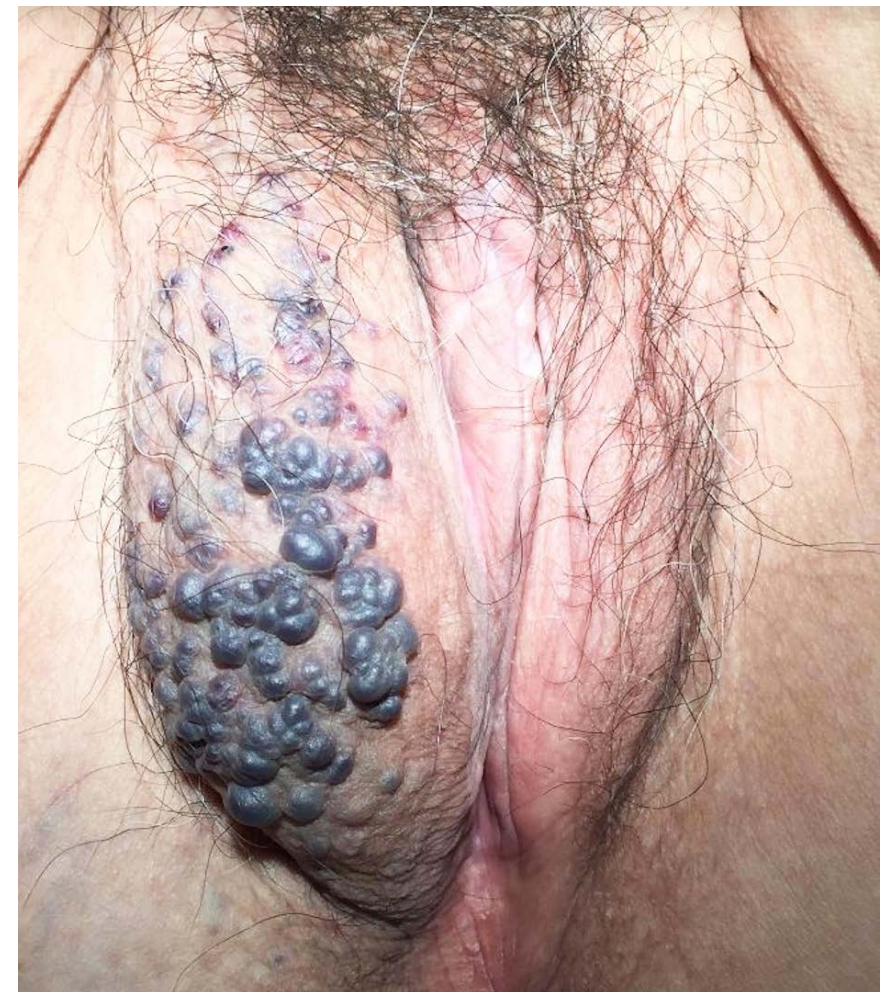

A

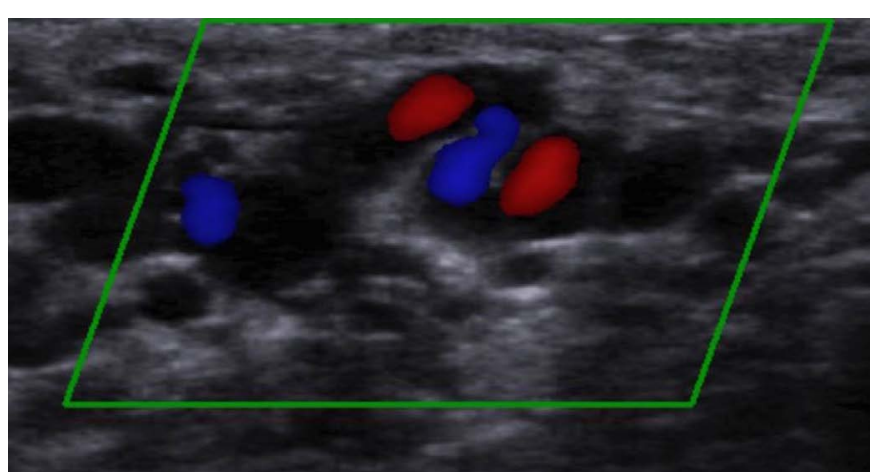

B

Fig. 6. A 63-yfar-old woman known to have vulvar hemangioma. A. Clinical image shows multiple purple-blıe swellings involving almost the whole of the right labia majora. B. Color Doppler ultrasonography shows tortuous vessels with multidirectional, turbulent blood flow, which also illustrates the extent of the lesion. 


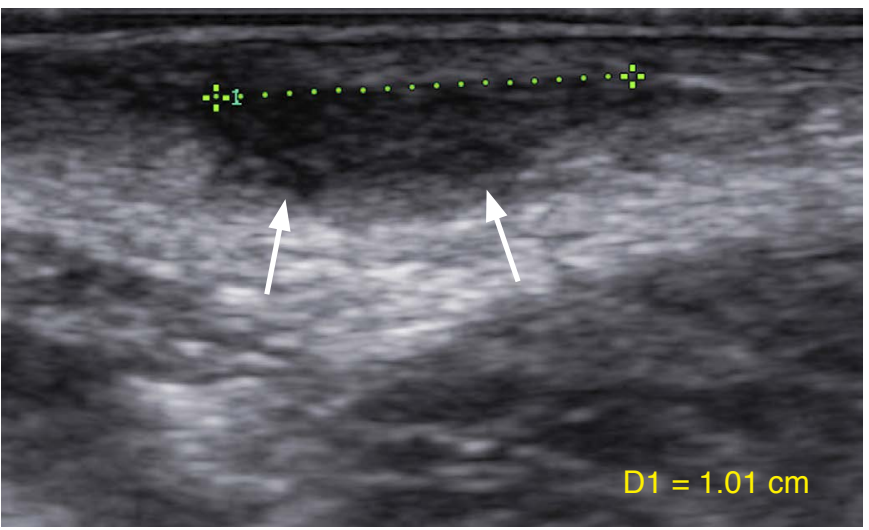

Fig. 7. A 55-year-old woman presented with a left vulvar nodule. Ultrasonography shows a $1 \times 1 \times 0.5-\mathrm{cm}$, ill-defined, nodular lesion (arrows) in the subcutaneous layer with mixed echogenicity comprising an echogenic hypocellular myxoid stroma interspersed with irregular hypoechoic areas of hypercellularity. Excisional biopsy confirmed angiomyofibroblastoma.

hemangiomas are commonly found in the skin and subcutaneous tissue. However, their occurrence in the female genital tract is rare [7]. Clinically, hemangiomas appear as multiple purple-blue swellings (Fig. 6A). Color Doppler ultrasound shows tortuous vessels with multidirectional, turbulent blood flow (Fig. 6B), which can also help to delineate the extent of the lesion.

\section{Angiomyofibroblastoma}

Angiomyofibroblastoma is a benign mesenchymal soft tissue tumor that occurs predominantly in the vulvar region of middleaged women. However, it can also occur in the vagina, cervix, and retroperitoneum [8]. It is a well-circumscribed, elastic, soft subcutaneous tumor and is usually less than $5 \mathrm{~cm}$ in diameter. Sonographically, it is described as a well-demarcated soft tissue mass with non-homogeneous mixed echogenicity, comprising an echogenic hypocellular myxoid stroma interspersed with irregular hypoechoic areas of hypercellularity (Fig. 7) [9]. Occasionally, multiple variable-sized cystic lesions within the mass can be seen, corresponding to dilated glands in the vulva. Color Doppler ultrasonography shows no vascularity within the mass [9].

\section{Soft Tissue Infection}

The distinction between vulvar abscess and cellulitis is important, because the treatment of these conditions is different. This condition is commonly seen in the emergency department where point-ofcare ultrasound may help in making the correct diagnosis [10]. Fig. 8 is a sonogram from a 31-year-old woman who presented with an approximately $1-\mathrm{cm}$ tender lesion over the right labia majora. Ultrasound favored the diagnosis of cellulitis rather than an abscess,

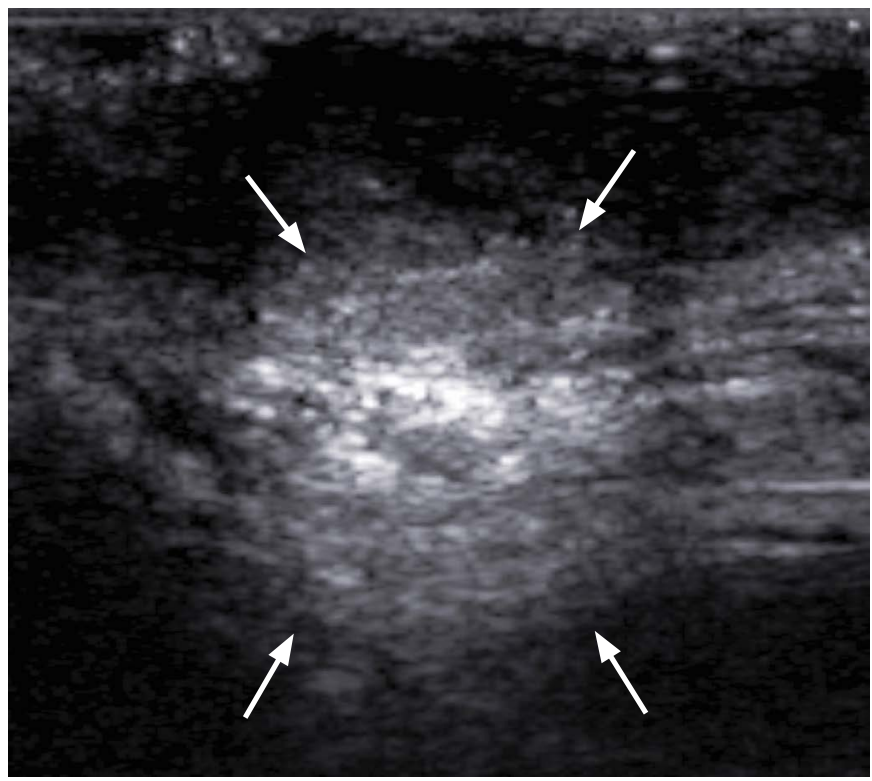

Fig. 8. A 31-year-old woman presented with an approximately $1-\mathrm{cm}$ tender lesion over the right labia majora. Ultrasonography shows a diffuse echogenic area with no discrete collection seen over the involved area; this favored the diagnosis of cellulitis, rather than an abscess. The lesion resolved after antibiotic therapy, with no need for drainage. The arrows outline the area of involvement.

and the lesion was therefore successfully treated with antibiotic therapy, without the need for drainage.

\section{Conclusion}

Many different dermatological and gynecological entities can occur on the vulva. The vulvar lesions that are described in this article only represent those encountered by the author, and this review is clearly not exhaustive. Ultrasonography can play a helpful role in the evaluation of benign vulvar lesions, particularly when unfamiliar lesions are encountered. Ultrasonography can assist in determining the location, depth of involvement (particularly in relation to the skin layers), exact size, contents, and vascularity of a lesion. It is also useful for monitoring such lesions when they are managed nonsurgically. Therefore, this review provides background information on the application of ultrasonography for the evaluation of vulvar lesions, with the inclusion of basic guidelines on the relevant techniques and normal ultrasonographic anatomy. This review promotes physicians' awareness of the ultrasonographic appearance of various vulvar conditions, with the goal of increasing physicians' interest in and utilization of ultrasonography for the evaluation of vulvar conditions.

ORCID: Vincent Y. T. Cheung: http://orcid.org/0000-0002-3293-0697 


\section{Conflict of Interest}

No potential conflict of interest relevant to this article was reported.

\section{References}

1. Wortsman X. Ultrasound in dermatology: why, how, and when? Semin Ultrasound CT MR 2013;34:177-195.

2. Wortsman X. Sonography of dermatologic emergencies. J Ultrasound Med 2017;36:1905-1914.

3. Barcaui Ede O, Carvalho AC, Pineiro-Maceira J, Barcaui CB, Moraes $\mathrm{H}$. Study of the skin anatomy with high-frequency (22 $\mathrm{MHz}$ ) ultrasonography and histological correlation. Radiol Bras 2015;48:324-329.

4. Eppel W, Frigo P, Worda C, Bettelheim D. Ultrasound imaging of Bartholin's cysts. Gynecol Obstet Invest 2000;49:179-182.
5. Abulafia O, Sherer DM. Bartholin gland abscess: sonographic findings. J Clin Ultrasound 1997;25:47-49.

6. Sherer DM, Gorelick C, Wagreich A, Lee YC, Serur E, Zigalo A, et al. Sonographic findings of a large vulvar lipoma. Ultrasound Obstet Gynecol 2007;30:786-787.

7. Cheung VYT, Tse KY. Vulval hemangioma. J Obstet Gynaecol Can 2017 May 4 [Epub]. https://doi.org/10.1016/j.jogc.2017.02.004.

8. Naheed S, Upadhyay K, Pradeep K. Angiomyofibroblastoma of the vulva. J Obstet Gynaecol 2011;31:554-555.

9. Kim SW, Lee JH, Han JK, Jeon S. Angiomyofibroblastoma of the vulva: sonographic and computed tomographic findings with pathologic correlation. J Ultrasound Med 2009;28:1417-1420.

10. Lin MJ, Neuman M, Rempell R, Monuteaux M, Levy J. Point-of-care ultrasound is associated with decreased length of stay in children presenting to the emergency department with soft tissue infection. J Emerg Med 2018;54:96-101. 